\title{
Associations between plasma branched-chain amino acids, $\beta$-aminoisobutyric acid and body composition
}

\author{
Annemarie Rietman ${ }^{1} *$, Takara L. Stanley ${ }^{2,3}$, Clary Clish ${ }^{4}$, Vamsi Mootha ${ }^{4,5}$, Marco Mensink ${ }^{1}$, \\ Steven K. Grinspoon ${ }^{2}$ and Hideo Makimura ${ }^{2}$ \\ ${ }^{1}$ Division of Human Nutrition, Wageningen University, NL-6703 HD Wageningen, The Netherlands \\ ${ }^{2}$ Program in Nutritional Metabolism and Neuroendocrine Unit, Massachusetts General Hospital and Harvard Medical School, Boston, \\ MA 02114, USA \\ ${ }^{3}$ Pediatric Endocrine Unit, Massachusetts General Hospital, Boston, MA 02114, USA \\ ${ }^{4}$ Broad Institute of MIT and Harvard, Cambridge, MA 02142, USA \\ ${ }^{5}$ Center for Human Genetic Research, Massachusetts General Hospital and Harvard Medical School, Boston, MA 02114, USA
}

(Received 11 June 2015 - Final revision received 4 November 2015 - Accepted 18 November 2015)

Journal of Nutritional Science (2016), vol. 5, e6, page 1 of 8

doi:10.1017/jns.2015.37

Abstract

Plasma branched-chain amino acids (BCAA) are elevated in obesity and associated with increased cardiometabolic risk. $\beta$-Aminoisobutyric acid (B-AIBA), a recently identified small molecule metabolite, is associated with decreased cardiometabolic risk. Therefore, we investigated the association of BCAA and BAIBA with each other and with detailed body composition parameters, including abdominal visceral adipose tissue (VAT) and subcutaneous adipose tissue (SAT). A cross-sectional study was carried out with lean $(n 15)$ and obese $(n 33)$ men and women. Detailed metabolic evaluations, including measures of body composition, insulin sensitivity and plasma metabolomics were completed. Plasma BCAA were higher $\left(1.6(\mathrm{SE} 0 \cdot 08)\left(\times 10^{7}\right) v .1 \cdot 3(\mathrm{SE} 0 \cdot 06)\left(\times 10^{7}\right)\right.$ arbitrary units; $P=0.005)$ in obese $v$. lean subjects. BCAA were positively associated with VAT $(\mathrm{R} 0 \cdot 49 ; P=0 \cdot 0006)$ and trended to an association with SAT (R $0 \cdot 29$; $P=0.052)$. The association between BCAA and VAT, but not SAT, remained significant after controlling for age, sex and race on multivariate modelling $(P$ $<0 \cdot 05$ ). BCAA were also associated with parameters of insulin sensitivity (Matsuda index: $R-0 \cdot 50, P=0.0004$; glucose AUC: $R \quad 0 \cdot 53, P<0 \cdot 001$ ). BCAA were not associated with B-AIBA $(R-0 \cdot 04 ; P=0.79)$. B-AIBA was negatively associated with SAT $(\mathrm{R}-0 \cdot 37 ; P=0.01)$ but only trended to an association with VAT $(R \quad 0 \cdot 27 ; P=0 \cdot 07)$. However, neither relationship remained significant after multivariate modelling $(P>0 \cdot 05)$. Plasma B-AIBA was associated with parameters of insulin sensitivity (Matsuda index $R \quad 0 \cdot 36, P=0 \cdot 01$; glucose AUC: $R-0 \cdot 30, P=0 \cdot 04$ ). Plasma BCAA levels were positively correlated with VAT and markers of insulin resistance. The results suggest a possible complex role of adipose tissue in BCAA homeostasis and insulin resistance.

Key words: Branched-chain amino acids: Visceral adiposity: $\beta$-Aminoisobutyric acid: Subcutaneous adipose tissue: Lean body mass: Metabolomics

Worldwide, the number of people suffering from obesity continues to increase $\mathrm{i}^{(1)}$. Obesity is characterised by increases in both the abdominal subcutaneous adipose tissue (SAT) and visceral adipose tissue (VAT). SAT is considered to have more protective properties in relation to cardiometabolic risk factors ${ }^{(2)}$, whereas VAT is detrimental to cardiometabolic health ${ }^{(3,4)}$.
Obesity is also associated with elevated levels of plasma branched-chain amino acids (BCAA) $)^{(5)}$. The BCAA valine, leucine and isoleucine are associated with insulin resistance $^{(6-11)}$. Acute increases in plasma amino acids worsen insulin sensitivity ${ }^{(12)}$, and higher levels of dietary protein intake is associated with impaired glucose metabolism ${ }^{(13)}$.

Abbreviations: AU, arbitrary units; B-AIBA, $\beta$-aminoisobutyric acid; BCAA, branched-chain amino acid; BCAT, branched-chain amino acid aminotransferase; BCKD, branched-chain $\alpha$-ketoacid dehydrogenase; DXA, dual-energy X-ray absorptiometry; HOMA-IR, homeostasis model assessment for insulin resistance; OGTT, oral glucose tolerance test; SAT, subcutaneous adipose tissue; VAT, visceral adipose tissue.

* Corresponding author: A. Rietman, email annemarierietman@hotmail.com 
Furthermore, levels of baseline circulating BCAA predicted the development of incident diabetes in a large longitudinal cohort study ${ }^{(11)}$ suggesting that the relationship between BCAA and insulin resistance may be causal. However, the relationship between BCAA and detailed parameters of body composition, specifically VAT, has yet to be reported.

$\beta$-Aminoisobutyric acid (B-AIBA), a small molecule metabolite, was recently identified using a metabolomics approach as a possible novel myokine that increases browning of white adipocytes in response to physical activity and was found to be inversely associated with cardiometabolic risk including fasting glucose, insulin and homeostasis model assessment (HOMA) in addition to TAG and cholesterol ${ }^{(14)}$. As B-AIBA can be formed by the catabolism of thymine and valine ${ }^{(14,15)}$ this may represent a possible pathway through which BCAA exert their metabolic effects. The catabolism of BCAA, valine in particular, could decrease circulating BCAA while increasing B-AIBA, both of which are associated with improved insulin resistance.

In the present study we investigated the association of BCAA and B-AIBA with each other and with detailed body composition parameters, including SAT and VAT, for the first time. We hypothesised that BCAA would be positively associated with VAT while B-AIBA would be negatively associated with VAT. This hypothesis was evaluated in a crosssectional study of lean and obese men and women for whom detailed metabolic evaluations were performed.

\section{Materials and methods}

\section{subjects}

A total of forty-eight lean (BMI $\left.<25 \mathrm{~kg} / \mathrm{m}^{2} ; n 15\right)$ and obese (BMI $\geq 30 \mathrm{~kg} / \mathrm{m}^{2} ; n$ 33) men and women from the Boston community were evaluated between November 2007 and March 2009 at the Massachusetts Institute of Technology and Massachusetts General Hospital Clinical Research Center. Subjects were between the ages of 18 and 55 years and were otherwise healthy. Subjects receiving anabolic steroids, glucocorticoids, testosterone, hormone replacement, hormonal contraception, growth hormone or medication for diabetes mellitus treatment were excluded. Subjects with a $\mathrm{Hb}$ level less than $110 \mathrm{~g} / \mathrm{l}$, creatinine above $15 \mathrm{mg} / \mathrm{l}$, aspartate aminotransferase more than $2 \cdot 5$-fold above the upper limit of normal, and chronic illness such as HIV were also excluded. Written informed consent was obtained from each subject before testing in accordance with the Committee on the Use of Humans as Experimental Subjects of the Massachusetts Institute of Technology and the Subcommittee on Human Studies at the Massachusetts General Hospital.

\section{Body composition analyses}

Anthropometric measurements including height, body weight, and waist and hip circumference were obtained in triplicate by a trained nutritionist after an overnight fast. Total body fat percentage was determined by dual-energy X-ray absorptiometry (DXA) testing using a Hologic-4500 densitometer (Hologic,
Inc.). DXA uses a three-compartment model, partitioning tissue into lean, bone and fat mass. We performed $1 \mathrm{~cm}$ crosssectional abdominal computed tomography (CT) scans at the level of L4 to assess the distribution of abdominal SAT and abdominal VAT as previously described ${ }^{(16)}$.

\section{Biochemical analyses}

Fasting blood samples were drawn and oral glucose tolerance tests (OGTT) were performed using the $75 \mathrm{~g}$ oral glucose challenge. Glucose and insulin were obtained at 0, 30, 60, 90 and $120 \mathrm{~min}$. Homeostasis model assessment for insulin resistance (HOMA-IR) and the Matsuda index ${ }^{(17)}$ were calculated using the following equations:

$$
\begin{array}{r}
\mathrm{HOMA}=(\text { fasting glucose }(\mathrm{mmol} / \mathrm{l}) \\
\quad \times \text { fasting insulin }(\mathrm{mIU} / \mathrm{l})) / 22 \cdot 5 \\
\operatorname{ISI}_{(\text {Matsuda })}=\frac{10000}{\sqrt{G_{0} \times I_{0} \times G_{\text {mean }} \times I_{\text {mean }}}}
\end{array}
$$

$G_{\text {mean }}$ and $I_{\text {mean }}$ were obtained from values at time points 0 , 30, 60, 90 and $120 \mathrm{~min}$.

Measurement of fasting cholesterol profile was performed on a separate visit. Glucose and lipid levels were determined using standard methodology in the Massachusetts Institute of Technology clinical laboratory. Insulin was measured by a paramagnetic-particle chemiluminescence immunoassay using the Beckman Access Immunoassay System (Beckman Coulter). The analytical sensitivity of the assay is $0.03 \mathrm{IU} / \mathrm{ml}$, and the precision is $3-5 \cdot 6 \%$.

\section{Metabolomic profiling}

Metabolomic profiling was performed using liquid chromatography tandem mass spectrometry (LC-MS) for sixty-five polar metabolites including BCAA and B-AIBA from fasting plasma samples ${ }^{(11,18)}$. Polar metabolites were analysed in the hydrophilic interaction liquid chromatography/negative ion MS mode using targeted multiple reaction monitoring MS scans for optimal analytical sensitivity. To create this targeted profiling method, declustering potentials and collision energies were optimised for each metabolite by infusion of reference standards. In this method, the range of analyte signals spans at least four orders of magnitude dynamic range. Results are output in arbitrary units (AU). In general, the CV for repeated analyses are inversely proportional to the magnitude of the instrument response. Median CV was determined using repeated analyses of a pooled plasma reference sample $(r 10)$, and was $4.3 \% ;>70 \%$ of metabolites had a $\mathrm{CV} \leq 10 \%$. The BCAA leucine, isoleucine and valine were evaluated individually and summed and evaluated as total BCAA.

\section{Dietary evaluation}

Absolute intake of macronutrients including carbohydrates, proteins and fat were assessed by collection of a 4-d food record facilitated by a trained research dietitian during direct 
interview. Data were analysed using Nutrition Database Systems for Research (NDSR) software with the NDSR 2008 (version 2; University of Minnesota, Minneapolis, MN).

\section{Statistical analyses}

All data are expressed as mean values with their standard errors. Normality of distribution was assessed using Shapiro-Wilk analyses. Parameters that were not normally distributed were $\log$ transformed before analyses. All metabolites from the metabolomics assay were log transformed before analyses. A targeted statistical analysis using Pearson univariate regression analysis was performed to determine the relationship of (individual) BCAA and B-AIBA with body composition and metabolic parameters. As a secondary analysis, a non-targeted metabolomics analysis was performed assessing the relationship of all sixty-five polar metabolites to various body composition parameters. Multivariate regression analysis was performed evaluating metabolomic parameters and body composition parameters that were significant on univariate analysis. For body composition parameters, the model controlled for age, sex and race. Additionally, to investigate whether a relationship between (individual) BCAA and VAT can be explained by insulin resistance, the Matsuda index was included in a multivariate model. For the metabolic parameters, the model included age, sex, race and BMI. For our primary end point assessing the relationship of (individual) BCAA with body composition parameters, $P$ values were considered significant if $P<0 \cdot 05$, as this was a targeted analysis focusing specifically on (individual) BCAA. For the nontargeted secondary analyses evaluating all metabolites, $P$ values were considered significant if $<0.00074$ for Bonferroni correction for multiple comparisons.

Statistical analyses were performed with SAS 9.2 (20022008; SAS Institute Inc.).

\section{Results}

\section{Subjects}

A total of forty-eight subjects were evaluated. Of the subjects, fifteen were lean and thirty-three were obese. Subject characteristics can be found in Table 1. Briefly, lean subjects were 44.2 (SE 2.5) years, with a BMI of $22.6(\mathrm{SE} 0.3) \mathrm{kg} / \mathrm{m}^{2}$ and a waist circumference of $80 \cdot 1$ (SE 2.1) cm, while obese subjects were 37.8 (SE 1.7) years, with a BMI of 35.3 (SE $0.8) \mathrm{kg} / \mathrm{m}^{2}$ and a waist circumference of $110 \cdot 8$ (SE $2 \cdot 0$ ) $\mathrm{cm}$. The level of total plasma BCAA in lean subjects was 1.3 (SE $0.06)\left(\times 10^{7}\right)$ AU v. $1.6($ se 0.08$)\left(\times 10^{7}\right)$ AU $(P=0.005)$ in obese subjects. The individual BCAA were also significantly different in lean $v$. obese subjects (isoleucine: 4.4 (SE 0.3) $\left(\times 10^{6}\right)$ AU v. 5.6 (SE 0.3) $\left(\times 10^{6}\right)$ AU, $P=0.006$; leucine: $5 \cdot 4$ (SE $0 \cdot 2)\left(\times 10^{6}\right)$ AU v. $6.5($ SE $0 \cdot 3)\left(\times 10^{6}\right)$ AU, $P=0.02$; valine: $3 \cdot 1$ (SE 0.2) $\left(\times 10^{6}\right)$ AU v. 4.1 (SE 0.2) $\left(\times 10^{6}\right)$ AU, $\left.P=0 \cdot 0006\right)$. Plasma B-AIBA levels in lean subjects were $2 \cdot 5$ (SE $0 \cdot 3)\left(\times 10^{4}\right)$ AU v. 1.9 (SE 0.2) $\left(\times 10^{4}\right)$ AU $(P=0.08)$ in obese subjects.

\section{Branched-chain amino acids and $\beta$-aminoisobutyric acid}

Individual and total BCAA were not associated with B-AIBA (isoleucine: $R-0.007, P=0.96$; leucine: $R-0.07, P=0.67$; valine: $R-0.05, P=0.74$; total BCAA: $R-0.04, P=0.79$ ).

\section{Branched-chain amino acids, $\beta$-aminoisobutyric acid, amino acids and measures of body composition}

Individual and total BCAA were significantly associated with BMI (all $P<0.05$ ), waist circumference (all $P<0.01$ ) and VAT (all $P<0.005$ ) as detailed in Table 2 and Fig. 1.

Valine was positively associated with SAT, and isoleucine and total BCAA trended to an association with SAT, but leucine was not significantly associated with SAT (Table 2 and

Table 1. Subject characteristics ( $n$ 48)

(Mean values with their standard errors)

\begin{tabular}{|c|c|c|c|c|c|}
\hline & \multicolumn{2}{|c|}{ Normal-weight $(n 15)$} & \multicolumn{2}{|c|}{ Obese ( $n$ 33) } & \multirow[b]{2}{*}{$P$} \\
\hline & Mean & SE & Mean & SE & \\
\hline Age (years) & $44 \cdot 2$ & 2.5 & $37 \cdot 8$ & 1.7 & 0.07 \\
\hline Sex (no. male) & \multicolumn{2}{|c|}{8} & \multicolumn{2}{|c|}{13} & 0.28 \\
\hline Race (\% Caucasian) & \multicolumn{2}{|c|}{37.5} & \multicolumn{2}{|c|}{62.5} & 0.22 \\
\hline $\operatorname{BMl}\left(\mathrm{kg} / \mathrm{m}^{2}\right)$ & $22 \cdot 6$ & 0.3 & $35 \cdot 3$ & 0.8 & $<0.0001$ \\
\hline Waist circumference $(\mathrm{cm})$ & $80 \cdot 1$ & $2 \cdot 1$ & $110 \cdot 8$ & $2 \cdot 0$ & $<0.0001$ \\
\hline VAT $\left(\mathrm{cm}^{2}\right)$ & 53.0 & 13.7 & $123 \cdot 7$ & $10 \cdot 3$ & $<0.0001$ \\
\hline SAT $\left(\mathrm{cm}^{2}\right)$ & $139 \cdot 4$ & $21 \cdot 1$ & 503.4 & 30.4 & $<0.0001$ \\
\hline HOMA-IR & 0.6 & 0.1 & $2 \cdot 3$ & 0.5 & $<0.0001$ \\
\hline Matsuda index & 15.5 & 1.8 & $7 \cdot 0$ & 1.0 & $<0.0001$ \\
\hline Glucose AUC during OGTT (mmol/l/xmin) $\left(\times 10^{4}\right)$ & 0.072 & 0.0033 & 0.089 & 0.0050 & 0.04 \\
\hline TAG $(\mathrm{mmol} / \mathrm{l})$ & 0.561 & 0.049 & 1.338 & 0.149 & $<0.0001$ \\
\hline $\mathrm{HDL}(\mathrm{mmol} / \mathrm{l})$ & 1.55 & 0.06 & 1.18 & 0.05 & $<0.0001$ \\
\hline $\mathrm{LDL}(\mathrm{mmol} / \mathrm{l})$ & 2.57 & 0.21 & 3.02 & 0.15 & 0.09 \\
\hline \multicolumn{6}{|l|}{ BCAA } \\
\hline Total BCAA (AU) $\left(\times 10^{7}\right)$ & 1.3 & 0.06 & 1.6 & 0.08 & 0.005 \\
\hline Isoleucine $(A U)\left(\times 10^{6}\right)$ & 4.4 & 0.3 & 5.6 & 0.3 & 0.006 \\
\hline Leucine $(A \cup)\left(\times 10^{6}\right)$ & 5.4 & 0.2 & 6.5 & 0.3 & 0.02 \\
\hline Valine $(A U)\left(\times 10^{6}\right)$ & $3 \cdot 1$ & 0.2 & $4 \cdot 1$ & 0.2 & 0.0006 \\
\hline B-AIBA (AU) $\left(\times 10^{4}\right)$ & 2.5 & 0.3 & 1.9 & 0.2 & 0.08 \\
\hline
\end{tabular}

VAT, visceral adipose tissue; SAT, subcutaneous adipose tissue; HOMA-IR, homeostasis model assessment for insulin resistance; OGTT, oral glucose tolerance test; BCAA, branched-chain amino acids; AU, arbitrary units; B-AIBA, $\beta$-aminoisobutyric acid. 
Table 2. Univariate regression analyses of body composition to individual branched-chain amino acids (BCAA) and $\beta$-aminoisobutyric acid (B-AIBA)

\begin{tabular}{|c|c|c|c|c|c|c|c|c|c|c|}
\hline & $\begin{array}{l}\text { Leucine } \\
\qquad(R)\end{array}$ & $\begin{array}{l}\text { Leucine } \\
\qquad(P)\end{array}$ & $\begin{array}{l}\text { Isoleucine } \\
\qquad(R)\end{array}$ & $\begin{array}{l}\text { Isoleucine } \\
\qquad(P)\end{array}$ & $\begin{array}{l}\text { Valine } \\
\qquad(R)\end{array}$ & $\begin{array}{l}\text { Valine } \\
\quad(P)\end{array}$ & $\begin{array}{c}\text { BCAA } \\
(R)\end{array}$ & $\begin{array}{c}\mathrm{BCAA} \\
(P)\end{array}$ & $\begin{array}{l}\text { B-AIBA } \\
\quad(R)\end{array}$ & $\begin{array}{l}\text { B-AIBA } \\
(P)\end{array}$ \\
\hline BMI & 0.32 & 0.02 & 0.4 & 0.005 & 0.53 & $0.0001^{*}$ & 0.42 & 0.003 & -0.28 & 0.054 \\
\hline $\begin{array}{l}\text { Waist } \\
\text { circumference }\end{array}$ & 0.41 & 0.004 & 0.5 & $0.0004^{\star}$ & 0.58 & $<0.0001^{*}$ & 0.5 & 0.0004 & -0.18 & 0.24 \\
\hline VAT (CT) & 0.44 & 0.002 & 0.5 & $0.0004^{\star}$ & 0.48 & $0.0007^{*}$ & 0.49 & 0.0006 & -0.27 & 0.07 \\
\hline SAT (CT) & 0.19 & 0.2 & 0.28 & 0.06 & 0.4 & 0.006 & 0.29 & 0.052 & -0.37 & 0.01 \\
\hline Total fat \% (DXA) & 0.01 & 0.93 & 0.1 & 0.52 & 0.2 & 0.18 & 0.1 & 0.53 & -0.41 & 0.004 \\
\hline Total lean \% (DXA) & -0.1 & 0.64 & -0.1 & 0.63 & -0.21 & 0.15 & -0.11 & 0.43 & 0.36 & 0.01 \\
\hline
\end{tabular}

VAT, visceral adipose tissue; CT, computed tomography; SAT, subcutaneous adipose tissue; DXA, dual-energy X-ray absorptiometry.

* Significantly associated.

Fig. 2). BCAA were not significantly associated with percentage body fat and percentage lean body mass (Table 2).

Multivariate modelling correcting for age, sex and race continued to demonstrate a significant relationship between total BCAA and waist circumference $(P=0.0004)$ and $\operatorname{VAT}(P=$ $0 \cdot 002)$, independent of the other factors. When correcting for total fat mass, age, sex and race the significant relationship between total BCAA and VAT $(P=0 \cdot 23)$ was no longer significant. Likewise, in multivariate modelling correcting for BMI, age, sex and race, the relationship between total BCAA and VAT was no longer significant $(P=0 \cdot 52)$. Furthermore, in multivariate modelling including both VAT and the Matsuda index VAT was no longer significant $(P=$ 0.61) while the relationship between the Matsuda index and BCAA remained significant $(P=0 \cdot 0001)$.

B-AIBA was significantly associated with SAT $(R-0 \cdot 37$; $P=0.01)$, percentage body fat $(R-0.41 ; P=0.004)$, total fat mass $(R-0.34 ; P=0.02)$ and percentage lean body mass $(R 0.36 ; P=0 \cdot 01)$, and trended to an association with BMI and VAT (both $P<0 \cdot 10$ ), but not waist circumference
(Figs 1 and 2 and Table 2). However, none of these relationships remained significant upon multivariate modelling correcting for age, sex and race.

\section{Branched-chain amino acids, $\beta$-aminoisobutyric acid, amino acids and indices of insulin sensitivity}

Individual and total BCAA were significantly associated with the HOMA-IR index (all $P<0 \cdot 005$ ), Matsuda index (all $P<$ 0.005 ) and glucose AUC during OGT'T (all $P<0.005$ ), as detailed on Table 3 and Fig. 3.

Multivariate modelling correcting for age, sex, race and BMI continued to demonstrate a significant relationship between total BCAA and the HOMA-IR index $(P=0.03)$. In this model, age and BMI also remained associated with BCAA (age: $P=0 \cdot 04$; BMI: $P=0 \cdot 006$ ). Multivariate modelling also demonstrated a significant relationship between total BCAA and the Matsuda index $(P=0 \cdot 01)$. Age and BMI also remained associated with BCAA in this model (age: $P=0.05$; BMI: $P=0 \cdot 004)$. In additional multivariate modelling glucose
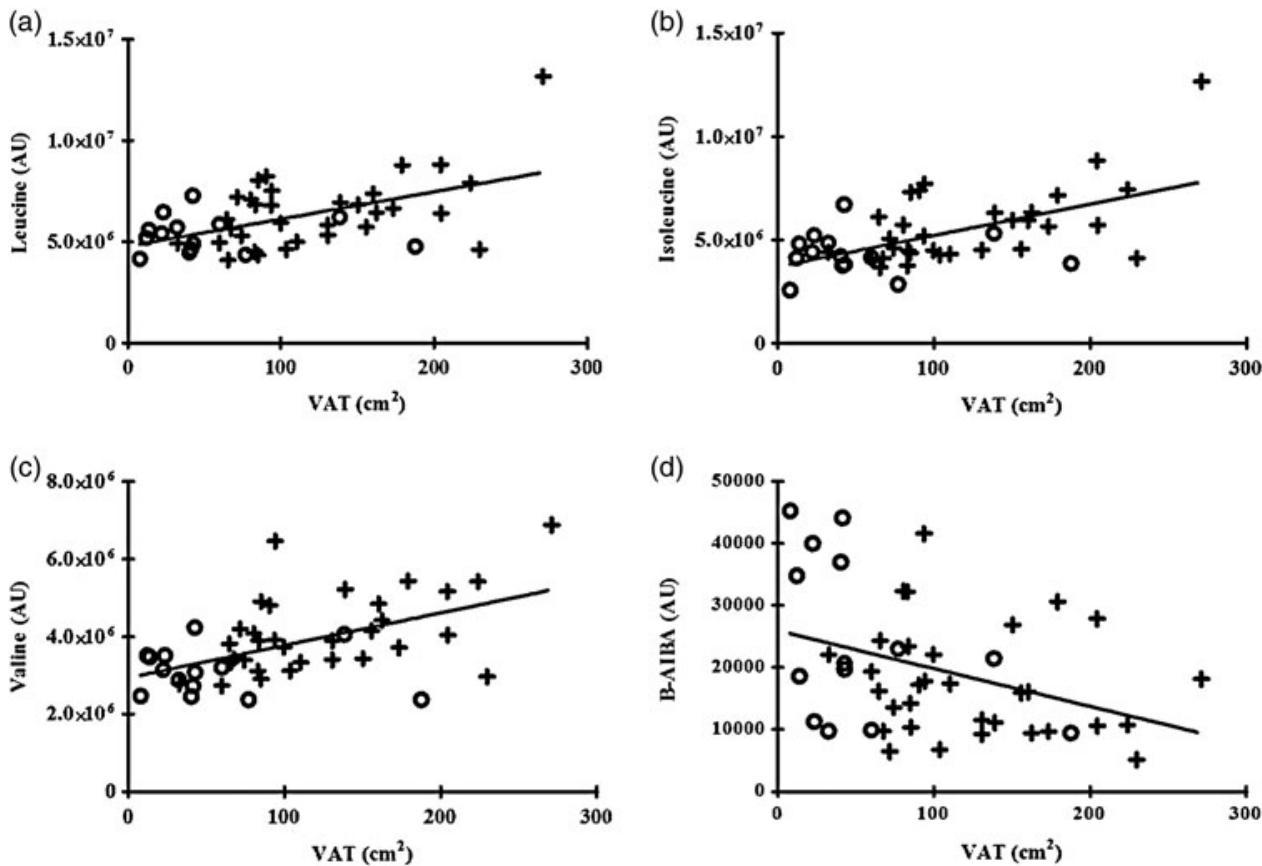

Fig. 1. Correlation between individual plasma branched-chain amino acids and $\beta$-aminoisobutyric acid (B-AIBA) $v$. visceral adipose tissue (VAT). (a) Leucine $v$. VAT; $R 0.44, P=0.002$. (b) Isoleucine $v$. VAT; $R 0.50, P=0.0004$. (c) Valine $v$. VAT; $R 0.48, P=0.0007$. (d) $B-A$ IBA $v$. VAT; $R-0.27, P=0.007$. $O$, Lean subjects; + , obese subjects; AU, arbitrary units. 

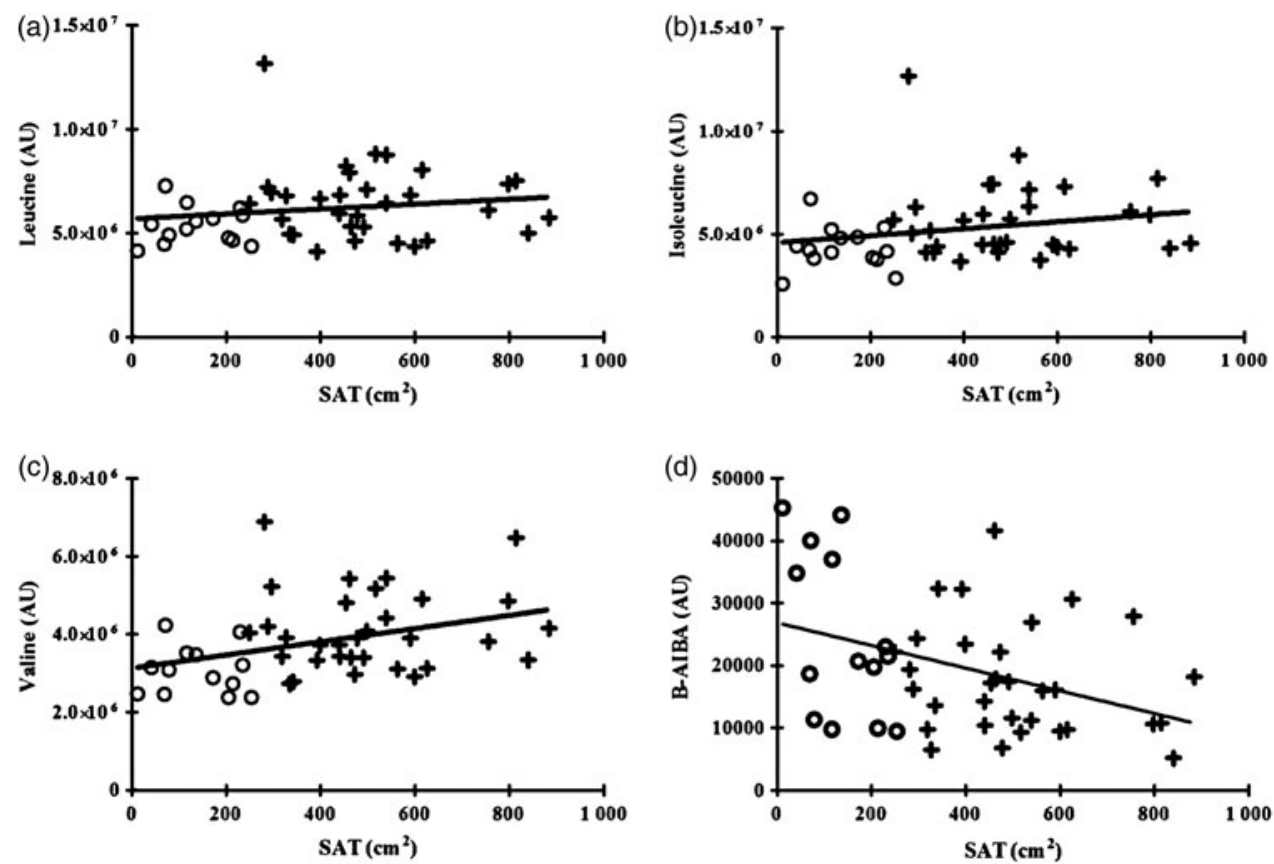

Fig. 2. Correlation between individual plasma branched-chain amino acids and $\beta$-aminoisobutyric acid (B-AIBA) $v$. subcutaneous adipose tissue (SAT). (a) Leucine v. SAT; $R$ 0.19, $P=0.20$. (b) Isoleucine $v$. SAT; $R 0.28, P=0.06$. (c) Valine $v$. SAT; $R 0.40, P=0.006$. (d) B-AIBA $v$. SAT; $R-0.37, P=0.01$. $O$, Lean subjects; + , obese subjects; $\mathrm{AU}$, arbitrary units.

AUC $(P=0.003)$ also remained significantly associated with BCAA independent of age, sex, race and BMI.

B-AIBA was negatively associated with the HOMA-IR index $(R-0.38 ; P=0.009)$ and glucose AUC during OGTT (R -0.30; $P=0.04$ ) and positively associated with the Matsuda index ( $R$ 0.36; $P=0 \cdot 01$ ) (Table 3 and Fig. 3). However, these associations no longer remained significant after multivariate modelling correcting for age, sex, race and BMI.

\section{Branched-chain amino acids, $\beta$-aminoisobutyric acid and dietary intake}

Neither plasma individual BCAA, nor total BCAA, nor B-AIBA were associated with absolute dietary protein, carbohydrate or fat intake (data not shown).

\section{Discussion}

In the present study, we demonstrated, for the first time, a significant association between individual and total BCAA and
VAT. We also confirmed the known association of BCAA with insulin resistance.

We showed, as hypothesised, a significant positive association between BCAA and VAT in this study. This suggests that adipose tissue may play an under-appreciated, but potentially significant, role in BCAA homeostasis ${ }^{(6,19)}$. The three $\mathrm{BCAA}$, valine, leucine and isoleucine, are essential amino acids and cannot be endogenously synthesised. As the dietary intake of proteins was not related to plasma BCAA levels in this study, the plasma levels of BCAA may primarily reflect inhibited catabolism of BCAA, as well as a decreased insulin sensitivity of the skeletal muscles leading to reduced inhibition of BCAA release by the skeletal muscle ${ }^{(7)}$. The catabolism of BCAA begins with the transport of BCAA into the cell, initiated by branched-chain amino acid aminotransferase (BCAT) in the mitochondrion ${ }^{(20)}$. There are two forms of BCAT: mitochondrial (BCATm) and a cytosolic (BCATc). BCATm is found in nearly all tissues, including adipose tissue ${ }^{(21,22)}$. BCAT catalyses reversible transamination of BCAA to form their $\alpha$-keto acids ${ }^{(23,24)}$. The second step of BCAA catabolism is irreversible oxidative decarboxylation,

Table 3. Univariate regression analyses of metabolic parameters to individual branched-chain amino acids (BCAA) and $\beta$-aminoisobutyric acid (B-AlBA)

\begin{tabular}{|c|c|c|c|c|c|c|c|c|c|c|}
\hline & $\begin{array}{l}\text { Leucine } \\
\qquad(R)\end{array}$ & $\begin{array}{l}\text { Leucine } \\
\qquad(P)\end{array}$ & $\begin{array}{l}\text { Isoleucine } \\
\qquad(R)\end{array}$ & $\begin{array}{l}\text { Isoleucine } \\
\qquad(P)\end{array}$ & $\begin{array}{l}\text { Valine } \\
\qquad(R)\end{array}$ & $\begin{array}{l}\text { Valine } \\
\qquad(P)\end{array}$ & $\begin{array}{c}\mathrm{BCAA} \\
(R)\end{array}$ & $\begin{array}{c}\text { BCAA } \\
(P)\end{array}$ & $\begin{array}{l}\text { B-AIBA } \\
\quad(R)\end{array}$ & $\begin{array}{l}\text { B-AIBA } \\
\quad(P)\end{array}$ \\
\hline HOMA-IR & 0.41 & 0.004 & 0.50 & 0.0003 & 0.47 & 0.0008 & 0.48 & 0.0007 & -0.38 & 0.009 \\
\hline Matsuda index & -0.44 & 0.002 & -0.50 & 0.0004 & -0.53 & 0.0002 & -0.50 & 0.0004 & 0.36 & 0.01 \\
\hline $\begin{array}{l}\text { Glucose AUC during } \\
\text { OGTT }\end{array}$ & 0.53 & 0.0001 & 0.50 & 0.004 & 0.50 & 0.0003 & 0.53 & 0.0001 & -0.30 & 0.04 \\
\hline TAG & 0.48 & 0.0006 & 0.50 & 0.0004 & 0.50 & 0.0003 & 0.51 & 0.0003 & -0.12 & 0.43 \\
\hline HDL & -0.42 & 0.003 & -0.38 & 0.009 & -0.46 & 0.001 & -0.43 & 0.003 & 0.21 & 0.16 \\
\hline LDL & 0.20 & 0.18 & 0.30 & 0.04 & 0.24 & 0.1 & 0.26 & 0.08 & -0.10 & 0.51 \\
\hline
\end{tabular}

HOMA-IR, homeostasis model assessment for insulin resistance; OGTT, oral glucose tolerance test. 
(a)

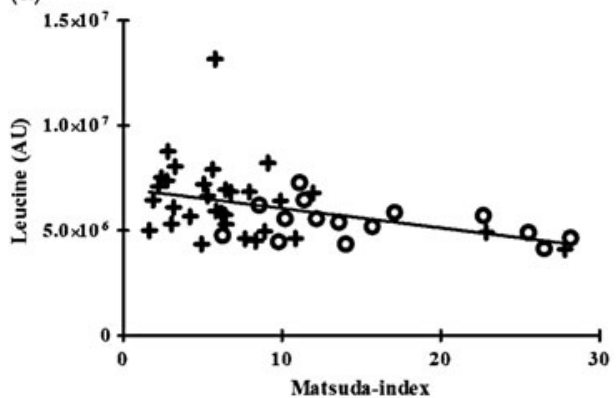

(c) 8

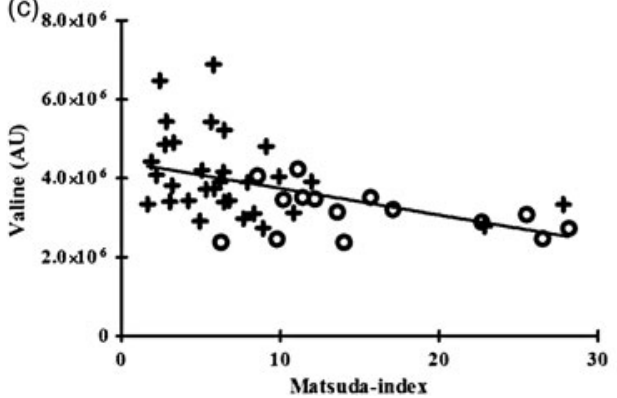

(b)

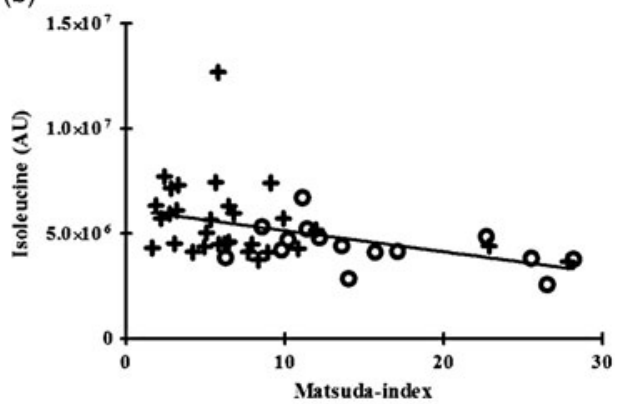

(d)

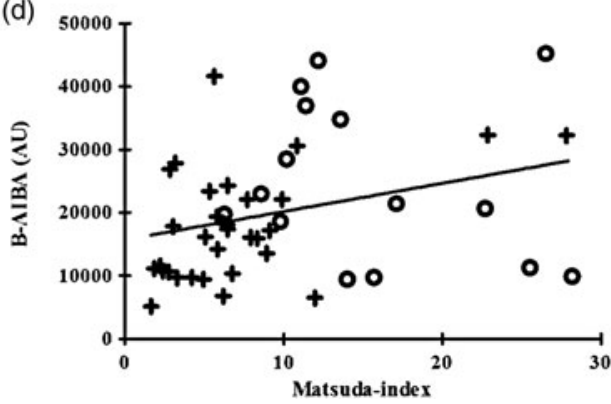

Fig. 3. Correlation between individual plasma branched-chain amino acids and $\beta$-aminoisobutyric acid (B-AIBA) $v$. Matsuda index. (a) Leucine $v$. Matsuda index; $R$ $-0.44, P=0.002$. (b) Isoleucine $v$. Matsuda index; $R-0.50, P=0.0004$. (c) Valine $v$. Matsuda index; $R-0.53, P=0.0002$. (d) B-AlBA $v$. Matsuda index; $R 0.36 ; P=$ 0.01 . $O$, Lean subjects; +, obese subjects; $\mathrm{AU}$, arbitrary units.

catalysed by the branched-chain $\alpha$-ketoacid dehydrogenase (BCKD) complex, which is located in the mitochondrial matrix $^{(20,23,24)}$. BCKD is the rate-limiting step in BCAA catabolism and its activity is decreased by increased acetyl-CoA concentration and NADH:NAD ${ }^{+}$ratio possibly due to $\beta$-oxidation of NEFA ${ }^{(\sigma)}$. Thus, increased NEFA present in obesity may decrease the activity of $\mathrm{BCKD}$, thereby decreasing the catabolism of BCAA.

The importance of adipose tissue in BCAA catabolism has been demonstrated in several studies. Protein levels of BCATm and BCKDE1, one of the three catalytic components of $\mathrm{BCKD}$, are reduced in $o b / o b$ mice, diet-induced obese mice and Zucker fatty rats ${ }^{(19,25)}$. Moreover, Herman et al. ${ }^{(21)}$ demonstrated that transplanting adipose tissue from wild-type littermates to BCAT2 knock-out mice can reduce circulating $\mathrm{BCAA}^{(21)}$. Human studies corroborate these findings. mRNA expression of BCKDHA, the human gene that encodes for the $\alpha$ subunit of E1 of BCKD, was reduced in adipocytes of obese insulin-resistant human subjects compared with lean subjects ${ }^{(19)}$. In addition, obese women with the metabolic syndrome have lower mRNA expression of BCKD in VAT as compared with healthy obese women without disturbed glucose metabolism ${ }^{(19)}$. Furthermore, obese patients who have undergone gastric bypass surgery to lose weight have increased levels of both BCATm and BCKDE1a mRNA expression in adipose tissue ${ }^{(25)}$. These studies and our study all support a significant metabolic role of adipose tissue in BCAA homeostasis.

We demonstrated a significant association between B-AIBA and percentage lean body mass. Although lean body mass as quantified by DXA consists of all non-fat, non-bone tissue, including but not limited to striated muscle, this association is consistent with the hypothesised role of B-AIBA as a muscle-derived metabolite. We also demonstrated a significant negative association between B-AIBA and total body fat percentage and SAT, although these relationships were no longer significant upon multivariate analyses. These results suggest that increased plasma B-AIBA concentration is associated with a more favourable body composition, e.g. more lean mass and more SAT $v$. VAT.

BCAA and B-AIBA were not significantly associated with each other in our study. While the lack of association does not necessarily rule out a direct relationship between BCAA and B-AIBA, it does suggest complexity in the generation and metabolism of B-AIBA and furthermore suggests that B-AIBA is not simply a breakdown product of valine ${ }^{(14,15)}$

Our results also confirmed the association between BCAA and markers of insulin sensitivity ${ }^{(5-7,9-11,26)}$ and lipid profile ${ }^{(27)}$ as previously demonstrated. The relationship of BCAA with HOMA-IR, the Matsuda index and glucose AUC after OGTT were strong and remained significant even after controlling for age, sex, race and BMI. Therefore, the role of insulin resistance in mediating the relationship between BCAA and VAT need to be considered. The addition of HOMA-IR or the Matsuda index to the multivariate regression model assessing the relationship between BCAA and VAT resulted in the loss of statistical significance between BCAA and VAT, suggesting that this relationship may be dependent upon insulin resistance.

In our study B-AIBA was not related to lipid parameters unlike a previous report ${ }^{(14)}$. Yet, B-AIBA did show an inverse relationship with markers of IR in our study.

Our study has some important limitations. First, we were not able to quantify the molar concentrations of BCAA and 
B-AIBA as no internal standard was used in the metabolite profiling. However, as the main focus of this investigation was the association between these metabolites and measures of body composition, the AU used are sufficient for that purpose and molar concentrations are not essential. Furthermore, with a relatively small sample size of forty-eight subjects, the study may not have been powered to detect true associations between BCAA, B-AIBA, and some measures of body composition or metabolism. Thus, lack of association in the present study should not be interpreted as absence of association.

The present study demonstrates an interesting relationship between BCAA and VAT. Shaham et ll $^{(18)}$ previously demonstrated that BCAA responses after OGTT remained higher in subjects with an impaired glucose tolerance compared with subjects with a normal glucose tolerance. This suggests that the inhibiting effect of insulin on proteolysis can be seen after an oral glucose challenge. Future research investigating the contribution of VAT to dynamic changes in BCAA in a post-absorptive state such as after an OGT'T may also be of interest. In addition, suppression of lipolysis has been suggested to be more sensitive to the actions of insulin compared with suppression of protein catabolism ${ }^{(18)}$. Given the possible role of NEFA in mediating BCAA catabolism ${ }^{(6)}$, assessing the contribution of fasting, nocturnal and post-absorptive NEFA in this context would also be warranted in future studies. Furthermore, consideration of the role of liver fat in BCAA metabolism may also be necessary in future studies given the known association between VAT and hepatic steatosis ${ }^{(4)}$ and the known role of the liver in amino acid metabolism.

To conclude, we demonstrate, for the first time, a significant positive relationship of plasma BCAA levels with VAT, which did not persist when adjusting for total fat mass, BMI or the Matsuda index. Furthermore, we once more showed a positive relationship of plasma BCAA and markers of insulin resistance. We also demonstrated a positive relationship of B-AIBA with lean body mass and a negative relationship with SAT and markers of insulin resistance. This study also adds further data supporting negative effects of VAT on cardiometabolic health. The results might suggest a more significant role of adipose tissue in BCAA homoeostasis than previously considered and indicate that further research in the field is needed. Furthermore, our results on B-AIBA and markers of cardiometabolic health together with other published studies ${ }^{(14,15)}$ suggest that further research into the regulation of this small molecule metabolite and its physiological significance is warranted.

\section{Supplementary material}

To view supplementary material for this article, please visit http://dx.doi.org/10.1017/jns.2015.37

\section{Acknowledgements}

A. R. is supported by the European Foundation for the Study of Diabetes (EFSD) Albert Renold travel award and the Global Dairy Platform. Additional support was provided by National Institutes of Health grant K23DK089910 to
T. L. S., R01HL085268 and P30DK040561 to S. K. G., K23DK087857 to H. M., and M01RR01066 and UL1RR025758, Harvard Clinical and Translational Science Center, from the National Center for Research Resources. The content is solely the responsibility of the authors and does not necessarily represent the official views of the National Center for Research Resources or the National Institutes of Health.

A. R. and T. L. S. analysed the data and performed statistical analyses. C. C. and V. M. contributed to the metabolomics analyses and interpretation. A. R. and H. M. wrote the manuscript. T. L. S., M. M. and S. K. G. contributed to manuscript preparation. H. M. designed the study, conducted experiments, and oversaw all aspects of the study, data interpretation and manuscript preparation.

The authors have nothing to disclose.

\section{References}

1. $\mathrm{Ng} \mathrm{M}$, Fleming $\mathrm{T}$, Robinson M, et al. (2014) Global, regional, and national prevalence of overweight and obesity in children and adults during 1980-2013: a systematic analysis for the Global Burden of Disease Study 2013. Lancet 384, 766-781.

2. Porter SA, Massaro JM, Hoffmann U, et al. (2009) Abdominal subcutaneous adipose tissue: a protective fat depot? Diabetes Care 32, 1068-1075.

3. Matsuzawa Y, Shimomura I, Nakamura T, et al. (1995) Pathophysiology and pathogenesis of visceral fat obesity. Obes Res 3, Suppl. 2, 187S-194S.

4. Tchernof A \& Després JP (2013) Pathophysiology of human visceral obesity: an update. Physiol Rev 93, 359-404.

5. Felig P, Marliss E \& Cahill GF Jr (1969) Plasma amino acid levels and insulin secretion in obesity. New Engl J Med 281, 811-816.

6. Adams SH (2011) Emerging perspectives on essential amino acid metabolism in obesity and the insulin-resistant state. Adv Nutr 2, 445-456.

7. Adeva MM, Calviño J, Souto G, et al. (2012) Insulin resistance and the metabolism of branched-chain amino acids in humans. Amino Acids 43, 171-181.

8. Cheng S, Rhee EP, Larson MG, et al. (2012) Metabolite profiling identifies pathways associated with metabolic risk in humans. Circulation 125, 2222-2231.

9. Newgard CB, An J, Bain JR, et al. (2009) A branched-chain amino acid-related metabolic signature that differentiates obese and lean humans and contributes to insulin resistance. Cell Metab 9, 565-566.

10. Tai ES, Tan MLS, Stevens RD, et al. (2010) Insulin resistance is associated with a metabolic profile of altered protein metabolism in Chinese and Asian-Indian men. Diabetologia 53, 757-767.

11. Wang TJ, Larson MG, Vasan RS, et al. (2011) Metabolite profiles and the risk of developing diabetes. Nat Med 17, 448-453.

12. Krebs M, Krssak M, Bernroider E, et al. (2002) Mechanism of amino acid-induced skeletal muscle insulin resistance in humans. Diabetes 51, 599-605.

13. Linn T, Santosa B, Grönemeyer D, et al. (2000) Effect of long-term dietary protein intake on glucose metabolism in humans. Diabetologia 43, 1257-1265.

14. Roberts LD, Boström P, O’Sullivan JF, et al. (2014) $\beta$-Aminoisobutyric acid induces browning of white fat and hepatic $\beta$-oxidation and is inversely correlated with cardiometabolic risk factors. Cell Metab 19, 96-108.

15. Begriche K, Massart J \& Fromenty B (2010) Effects of $\beta$-aminoisobutyric acid on leptin production and lipid homeostasis: mechanisms and possible relevance for the prevention of obesity. Fundam Clin Pharmacol 24, 269-282. 
16. Borkan GA, Gerzof SG, Robbins AH, et al. (1982) Assessment of abdominal fat content by computed tomography. Am J Clin Nutr 36, 172-177.

17. Matsuda M \& DeFronzo RA (1999) Insulin sensitivity indices obtained from oral glucose tolerance testing: comparison with the euglycemic insulin clamp. Diabetes Care 22, 1462-1470.

18. Shaham O, Wei R, Wang TJ, et al. (2008) Metabolic profiling of the human response to a glucose challenge reveals distinct axes of insulin sensitivity. Mol Syst Biol 4, 214.

19. Lackey DE, Lynch CJ, Olson KC, et al. (2013) Regulation of adipose branched-chain amino acid catabolism enzyme expression and cross-adipose amino acid flux in human obesity. Am J Physiol Endocrinol Metab 304, E1175-E1187.

20. Hutson SM, Sweatt AJ \& LaNoue KF (2005) Branched-chain amino acid metabolism: implications for establishing safe intakes. J Nutr 135, 1557S-1564S.

21. Herman MA, She P, Peroni OD, et al. (2010) Adipose tissue branched chain amino acid (BCAA) metabolism modulates circulating BCAA levels. J Biol Chem 285, 11348-11356.
22. Sitryawan A, Hawes JW, Harris RA, et al. (1998) A molecular model of human branched-chain amino acid metabolism. Am J Clin Nutr 68, 72-81.

23. Brosnan JT \& Brosnan ME (2006) Branched-chain amino acids: enzyme and substrate regulation. J Nutr 136, 207S-211S.

24. Islam MM, Wallin R, Wynn RM, et al. (2007) A novel branched-chain amino acid metabolon. Protein-protein interactions in a supramolecular complex. J Biol Chem 282, 1189311903.

25. She P, Van Horn C, Reid T, et al. (2007) Obesity-related elevations in plasma leucine are associated with alterations in enzymes involved in branched-chain amino acid metabolism. Am J Physiol Endocrinol Metab 293, E1552-E1563.

26. Shah SH, Crosslin DR, Haynes CS, et al. (2012) Branched-chain amino acid levels are associated with improvement in insulin resistance with weight loss. Diabetologia 55, 321-330.

27. Kamaura M, Nishijima K, Takahashi M, et al. (2010) Lifestyle modification in metabolic syndrome and associated changes in plasma amino acid profiles. Circ J 74, 2434-2440. 